(C) 2006 IEEE. Personal use of this material is permitted. Permission from IEEE must be obtained for all other uses, in any current or future media, including reprinting/republishing this material for advertising or promotional purposes, creating new collective works, for resale or redistribution to servers or lists, or reuse of any copyrighted component of this work in other works. 


\title{
The Design of a Fuel-Cell-Based Power-Quality Control Center to Realize Unbundled Power-Quality Supply
}

\author{
Y. Q. Zhan, Y. H. Li, S. S. Choi, S. Rajakaruna, and D. Mahinda Vilathgamuwa
}

\begin{abstract}
The application of a power-quality control center (PQCC) to provide an unbundled quality of electricity supply is examined. The center consists of back-to-back power converters, a dc bus and a solid-oxide fuel-cell distributed generator (SOFCDG). The purpose is to use the flexibility offered by the converters and SOFCDG to realize varied levels of ac supply quality to the Ioads. Through the concept of a feasible operating area of the SOFCDG, analysis shows that the ability of the POCC to accommodate instantaneous power demand change is constrained. This is due to limits imposed on the fuel utilization factor of the SOFCDG. A feasible PQCC operating scheme is then proposed. It fully exploits the ability of the converters and the distributed generator to respond to external disturbances. In the event of a system voltage disturbance, the $\mathrm{PQCC}$ is to operate in one of two scenarios in accordance to the severity of the disturbance. Based on the dynamic model derived for the power network, a design procedure of the PQCC control system is also described. Simulation results show the efficacy of the proposed scheme.
\end{abstract}

Index Terms-Distributed generator (DG), fuel cell (FC), powerquality control center (PQCC), unbundled PQ supply, voltage sag.

\section{INTRODUCTION}

I N MANY parts of the world, the design and operation of power systems would have to be re-examined in the light of the deregulation of their utility industry. Introducing small-scale power generation on the customer side of distribution systems would become more viable as the new paradigm allows the distributed generators (DGs) easier access to the transmission-distribution facilities [1]. At the load end, meanwhile, the trend is toward the increasingly widespread use of computer-controlled and automated equipment. These loads require a high level of supply quality. Thus, it is envisaged that the installation of the DG that is electrically closer to these sensitive loads would also lead to improved supply quality and reliability. Unfortunately, the introduction of DG brings with it some technical challenges as well. For example, the DG can cause power flows along unintended paths under certain operational conditions. This could result in an undesirable system voltage profile, or cause an increase in the fault level and the obstructions to fault detection, among other unwelcome consequences [2]. Furthermore, while

Manuscript received January 26, 2005: revised July 19. 2005. Paper no. TPWRD-0)(045-2005.

The authors are with the School of Electrical and Flectronic Engineering. Nanyang Technological University. Singapore 639798 (e-mail: esschoi (a) ntu.edu.sg).

Digital Object Identifier 10.1109/TPWRD.2005.8613.35 those automated or computer-controlled loads in a modern electricity network require a high-quality power supply, there is still a significant portion of the loads that can accept a lower-quality supply but demand it to be supplied at lower prices. In this regard, an unbundled power-quality (PQ) supply is therefore a highly desirable feature for the distribution systems of the future, for it then enables the utility industry to deliver different PQ levels to match customer requirements and expectations [3]. For example, one could envisage a number of feeders emanating from a distribution substation and with each feeder providing a specific level of $P Q$. A wafer-fabrication manufacturing concern could be served by feeder(s) designed to deliver the highest PQ while domestic load customers are more likely to opt for a lower quality of supply served by different feeder(s). An appropriate tariff structure could then be designed which will have tariff rates commensurate with the quality levels.

In order to meet the above requirements, several conceptual power distribution systems have been proposed. The power-quality control center (PQCC) [4] is one such possible design, which could be particularly suited to supply an industrial/commercial park where customers in close proximity require varied quality in a power supply. Indeed, in [5]. the authors describe the results of some preliminary works concerning a so-called premium PQ park. The park could be considered as a first step toward the development of a PQCC. In general, a PQCC attempts to overcome some of the technical challenges caused by the introduction of the DG while, at the same time, providing unbundled PQ services. The PQCC is located between the upstream transmission-distribution power system and the loads. Within the PQCC, power-electronic converters are used for increased Hexibility in controlling power flows to/from the upstream system while the DG performs the duties of efficient generation of the balance of the energy needs and acts as the backup energy source, among other functions. In [4], [6], and [7], several possible designs of the PQCC and the corresponding operational schemes have been suggested. One common feature of the designs is that they offer some degrees of flexibility in terms of the operation of the distribution systems. Notwithstanding these interesting developments, one also notices that there are still a number of technical issues that warrant deeper investigation. For instance, as the DG would play a central role in the PQCC operation, the unavoidable interactions between the DG and the upstream system should be carefully examined. Moreover, the authors of [4], [6], and [7] have not considered the impacts of the upstream or downstream fault on the PQCC in terms of the manner by which the DG 


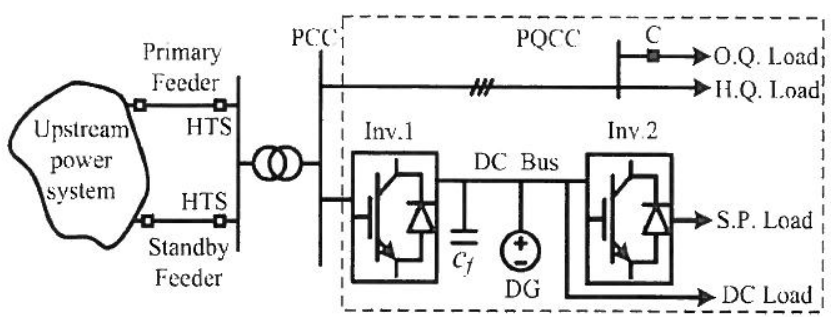

Fig. 1. Schematic diagram of the UPS-type PQCC.

and the power converters are controlled so as to maximize the potential benefits that can be extracted from the flexible distribution system.

Based on the work of [7], this paper presents an alternative PQCC scheme under both normal steady-state and upstream system fault conditions. The new scheme also strives for unbundled PQ supply by taking advantage of the flexibility offered by the PQCC, while certain operational constraints on the DG will be included. This is described in Section II. Section III describes the proposed PQCC operational scheme under steady-state as well as fault-contingency conditions. A brief description of the design of the control system for the PQCC system is then given in Section IV. Illustrative examples and simulation results are shown in Section V.

\section{SOME: BASIC CONSIDERATIONS}

Among the various types of PQCC configurations described in [6] and [7], the uninterruptible power supply (UPS)-type PQCC is selected for further analysis and design in this paper due to its flexibility and simplicity. In this section, a brief description of the PQCC and the power-generating capability of the DG are presented.

\section{A. UPS-Type PQCC Operational Scheme}

The schematic diagram of the UPS-type PQCC as proposed in [7] is shown in Fig. 1. It consists of two pulsewidth-modulated (PWM)-controlled inverters (Inv.1 and Inv.2) and a do bus. Unlike a conventional UPS system, however, the PQCC is equipped with a generating source in the form of a DG instead of batteries on the dc bus. Under such an arrangement, continuous power generation is possible as long as fuel supply to the DG is available. The upstream power system is interconnected to the PQCC through two feeders fitted with hybrid transfer switches (HTSs). Each HTS consists of a pair of antiparalleled thyristors and a mechanical switch. Detailed configuration and operation of HTSs can be found in [6]. As voltage transformation is envisaged between the upstream power system and the PQCC, a step-down transformer is shown connecting the upstream system and the point of common coupling (PCC) bus of the PQCC to reflect this requirement conceptually.

With this arrangement, there are three levels of PQ for the ac loads, named ordinary quality (OQ), high quality (HQ), and super premium quality (SP), in addition to one quality level for dc loads. Under normal steady-state, the upstream system supplies the entire OQ and HQ loads, and part of the SP and dc loads through only one of the two feeders, called the primary feeder. Although the exact form of the DG has not been reported in [6] and [7], the DG is expected to meet the remaining SP and do load demands.

Reference [7] proposes that should a fault occur on the primary feeder, its HTSs are turned off to isolate the fault from the PQCC and the HTSs on the standby feeder are turned on. The PQCC is then supplied by the standby feeder. Also, over the interval when the power transfer is switched from the primary to the standby feeders, the OQ load would be disconnected by the fast-acting circuit breaker (CB) $\mathrm{C}$ shown in Fig. 1. Any shortfall in meeting the HQ, SP, and dc load demands due to the delay in the switch-over transfer has to be met by the DG. In adopting this operating scheme. however, it is important to first consider the dynamic response capability of the DG. Depending on the relative sizes of the HQ. SP, and de loads in comparison with the DG capacity, the DG output power would have to change rapidly and at the precise output level when the upstream power system transits from a healthy into a faulted state. In the reported work, it is not clear to what extent the DG can accommodate such a power change while maintaining the desired supply quality. Second, it is clear that this arrangement would only cater to faults that occur on one of the feeders. In practice, the number of fault incidents on the directly connected feeder will be negligible compared to that originating on the much more extensive upstream power system. Hence, it is doubtful whether the standby feeder-HTS scheme would be a cost-effective proposition in practice.

In view of the above, the present investigation re-examines the work reported in [6] and [7], with the view to propose a more realistic scheme for the UPS-type PQCC under both steady-state and system fault conditions.

\section{B. Solid-Oxide Fuel-Cell-Distributed Generator}

In recent years, fuel cells (FCs) have emerged as attractive power sources since they are energy efficient, friendly to the environment and can provide reliable service [8]. [9]. While there are various kinds of FCs, the high-temperature solid-oxide fuel-cell (SOFC) power plant has been chosen as the form of DG in the present work. The main reason for doing so is because of the impressive service record of a number of demonstration units of SOFCDG currently in operation. SOFCDG can make use of hydrogen-rich natural gas as fuel infeed. It converts the chemical energy directly into dc electrical energy [10]. Although the energy conversion process is highly complex for the purpose of the present investigation, it would be sufficient to consider the schematic diagram of the SOFCDG shown in Fig. $2[11],[12]$. Two main parts of the DG can be readily identified. The part called the balance of plant (BOP) consists of the natural gas fuel storage, fuel valve, and the fuel processor. The fuel processor reforms the natural gas, of teh input flow rate $N_{f}$, to the hydrogen-rich fuel with a flow rate of $N_{H_{2}}^{\text {in }}$. The processor dynamic can be approximated by a first-order lag model of time constant $\tau_{f}$ [12]. The part labeled as the FC stack is where the complex thermodynamic and electrochemical processes take place. Details are given in [10].

On the electrical characteristics, the SOFC terminal voltage $V_{\text {d. c can be expressed as }}$

$$
V_{\mathrm{dc}}=E-r I_{\mathrm{FC}}
$$




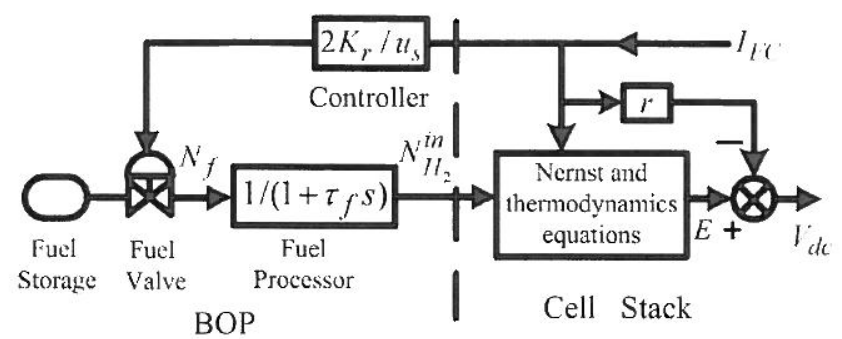

Fig. 2. Schematic diagram of an S(OFCDG

where $E$ is the internal electromotive force (emf) and $I_{1 \times} \cdot$ is the FC stack current [12]. Within the stack, there are the so-called ohmic, activation, and concentration losses. In the model, $r$ accounts for the losses. The calculation of $E$ is through the application of the well-known Nernst equation [10]. The expression is a highly nonlinear function of the hydrogen, oxygen, and water partial pressures and cells operating temperature. The electrochemical process to generate $E$ is shown simply in Fig. 2 by the block "Nernst and thermodynamics equations" where the input variables are $N_{H_{2}}^{\mathrm{in}}$ and $I_{\mathrm{F}} \cdot l \cdot k$, therefore, depends essentially on the SOFCDG internal electrochemical dynamics. As can be seen in [11] and [12], a change in the stack current $I_{\mathrm{F}}$, will typically take tens of seconds for its effect to be fully felt in $\mathrm{E}$. In the context of the problem inhand. however. one of the main considerations in the design of the PQCC is to mitigate the effects of upstream voltage-sag event on loads [7]. Often, the voltage sag is caused by the occurrence of a short-circuit fault in the upstream system. The duration of the sag is typically of the order of $I s$ or less. Hence, $E$ can be assumed to be essentially constant under such a short-duration disturbance condition and (1) can be used to describe the circuit behavior of the DG. Furthermore. the SOFCDG output power $\left(P_{d y}\right)$ is then given by

$$
P_{d y}=V_{\mathrm{dc}} I_{\mathrm{Fc}} .
$$

Next, the operation of SOFCDG has to take into account two practical constraints. The first pertains to the fuel utilization factor $u$. It is a most important operating variable affecting the performance of SOFCDG and is defined as

$$
u \equiv \frac{\left(N_{H_{2}}^{\mathrm{in}}-N_{H_{2}}^{\prime \prime}\right)}{N_{H_{2}}^{\mathrm{in}}}
$$

where $N_{H_{z}}^{\mathrm{in}}$ and $N_{H_{z}}^{\prime \prime}$ are the input and output flow rates of hydrogen in the FC stack. It is shown in [12] that $u$ can also be expressed in terms of $I_{\mathrm{FC}}$ as

$$
u=\frac{2 K_{r} I_{\mathrm{FC}}}{N_{H_{2}}^{\mathrm{in}}}
$$

where $K_{r}$ is a modeling parameter. The typical desirable range of $u$, expressed as $u_{\min } \leq u \leq u_{\text {max }}$, is from 0.7 to 0.9 . The overused-fuel condition $(u>0.9)$ could lead to permanent damage to the cells due to fuel starvation whereas the underused-fuel situation $(u<0.7)$ results in undesirably high cell voltages.

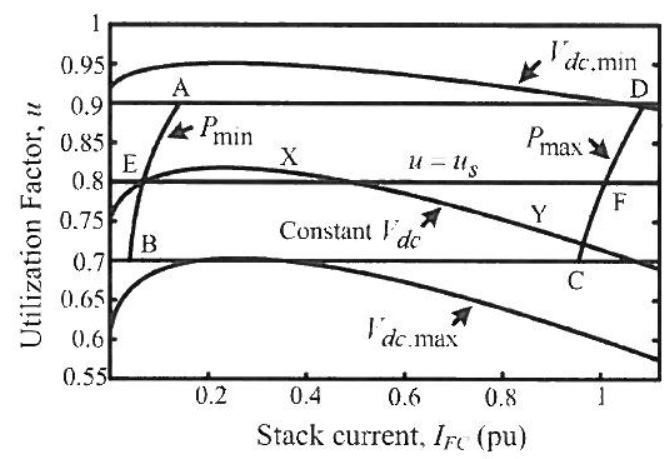

Fig. 3, Feasible operation area of an S()FCDG

The second constraint concerns the FC output power $I_{t l y}$. It should be maintained within the range

$$
P_{\min } \leq I_{d y}^{\prime} \leq I_{\text {minx }}^{\prime}
$$

The minimum power constraint is necessary in order to maintain stable stack operating temperature and acceptable power plant efficiency $[13]$. $P_{\text {max }}$ denotes the design power rating of the SOFCDG.

Due to the above considerations, Fig. 3 shows the steady-state feasible operating area (FOA) of the SOFCDG derived using the typical data given in [12]. The operating temperature of the FC stack is assumed constant in deriving the FOA, and the detailed construction of the FOA has been described in $|11|$. In arriving at this diagram, the FC-rated power and output voltage are used as the base quantities. The constraints placed on $u$ are represented by the horizontal lines $\mathrm{AD}$ and $\mathrm{BC}$. The limits on $P_{d t !}$ are shown by the curves $A B$ and $C D$. The FOA of the SOFCDG would correspond to the area "ABCDA." The SOFCDG must operate within the FOA as an operation that is outside FOA will reduce cell life and is unacceptable. Also shown in Fig. 3 is the curve $X Y$, describing the operational characteristic of the SOFCDG under a constant $V_{\text {dc }}$ condition. It shows $V_{\text {lc }}$ operating within the voltage limits $V_{d c, \text { max }}$ and $V_{d r, m i n}$.

According to (3), if one were to operate the FC stack with a fuel input $\left(N_{H_{2}}^{\text {in }}\right)$ proportional to $I_{\mathrm{Fc}}$, as governed by

$$
N_{f}=\frac{2 K_{r} I_{\mathrm{FC}}}{u_{s}}
$$

it will result in aconstant utilization factor $u_{s}$ under steady-state. Such an input fuel-control scheme has been incorporated in Fig. 2. Notice that by adopting a constant $u$ operational scheme. such as that described by the line EF in Fig. 3, $V_{\mathrm{d}}$. would vary as $I_{\mathrm{F} C}$ changes.

In order to ensure that cell operation will remain within the FOA during power system disturbances, one needs to determine the maximum step change in $I_{\mathrm{F}} \cdot$ that the SOFCDG can accommodate. Based on (3), a step change of $I_{\mathrm{F} \text { ( }}$ can be related to a step change in $u$ as

$$
\Delta I_{\mathrm{FC}}=\left(\frac{N_{H_{2}}^{\mathrm{III}}}{2 K_{r}}\right) \cdot \Delta u
$$


where the hydrogen input $N_{H_{2}}^{i n}$ will remain constant following the sudden step disturbance. This is due to the time lag in the fuel processor. In what follows, the subscript "()" would be used to denote the initial value of the respective variable. Hence, the initial operating current and utilization factor are $I_{\mathrm{Fc}, 0}$ and $u_{s}$. respectively. It can be deduced that

$$
\frac{\Delta I_{\mathrm{FC}}}{I_{\mathrm{I} \cdot(\cdot, 0}}=\frac{\Delta u}{u_{s}}
$$

Since the acceptable range of $u$ is from $u_{\min }$ to $u_{\text {max }}$, one can define a new variable $z$, where

$$
\varepsilon= \begin{cases}\frac{\left(u_{n+\ldots}-u\right)}{u}, & \text { for positive } \mathrm{I}_{\mathrm{I},} \cdot \text { step } \\ \frac{\left(u_{\min }-u_{s}\right)}{u}, & \text { for negative } \mathrm{I}_{\mathrm{I} \cdot \mathrm{c}} \text { step. }\end{cases}
$$

Therefore, the maximum step change in $J_{\mathrm{IC}}$, which can be accommodated by the SOFCDG is given by

$$
\Delta I_{\mathrm{F}(} \cdot \leq|\varepsilon| I_{\mathrm{F}(\cdot, 0)}
$$

By neglecting the small voltage drop due to the ohmic loss in (1) and then using (2), the maximum step change in the SOFCDG power output while constrained by the FOA can be written as

$$
\Delta I_{d ! q}^{\prime} \leq|\varepsilon| I_{d q, 11}^{\prime}
$$

where $P_{t g, 01}=V_{d k \cdot, t)} I_{\mathrm{I}\left(c_{0}, 0\right.}$ is the initial operating power level.

For a power change larger than that given by (10), however. the SOFCDG would require several tens of seconds for the fuel processor to adjust the fuel input [12] if the cell is to be constrained to operate within the FOA.

In summary, it is clear that there is a practical limit in terms of the magnitude and the speed by which the DG can respond to a power change demand. Due to the inherent slow dynamics of the SOFCDG, a buffer energy storage device, such as a supercapacitor or battery, is often used in conjunction with the SOFCDG system [14], [15]. In the next section, however, an alternative scheme is proposed in which the buffer energy storage device would not be included. Instead, the intention is to investigate to what extent the PQCC can meet the need of providing an unbundled PQ supply for loads.

\section{Proposed SCHEME:}

Recognizing the shortcomings and practical problems faced in implementing the PQCC scheme described in [7], a new scheme is now proposed. The design is based on the consideration of normal system conditions as well as that pertaining to the upstream faults or outages of the various components in the power system.

\section{A. Steadr-State Operation Under Normal Conditions}

To facilitate the description of the scheme, the simplified equivalent circuit PQCC model shown in Fig. 4 is used. The standby feeder and its HTS used in [7] have been excluded in the present proposal. The upstream system is represented simply as an equivalent voltage source with its equivalent series

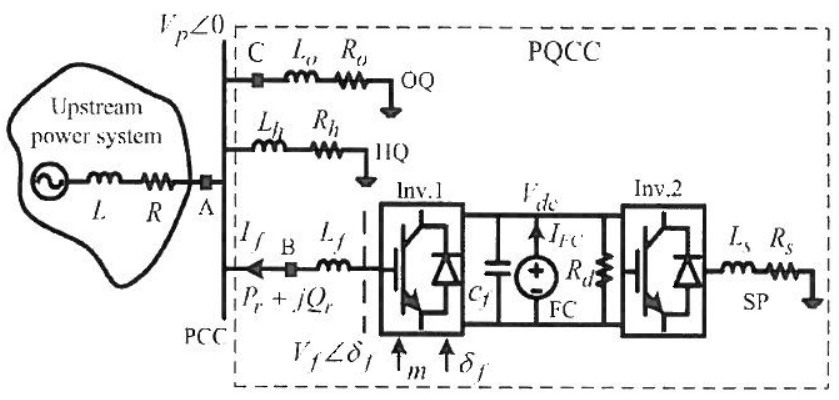

Fig. 4. Simplified PQCC model.

impedance $R+j w L$, where $w$ is the ac system frequency. In this figure, the ac voltages are measured phase-ground whereas the power flows are three-phase quantities. The PCC voltage phasor is treated as the reference quantity and is denoted as $\vec{V}_{p}, \vec{V}_{p}$ is assumed measurable. The SP, de, HQ, and OQ loads are represented by the respective impedances for convenience although in the analysis to follow, the load model used is a more general static-type. $L_{f}$ represents the total inductance which links the PCC to Inv.1. It also acts as a filter for high-frequency harmonics produced by the inverter and, therefore, the inverter harmonics are ignored in the following analysis. Although the SOFCDG in Fig. 4 is shown as a dc source, its functional characteristics described in Section II-B are included in the analysis. The capacitor $C_{f}$ shown connected to the dc bus is only used for filtering purposes and is not sufficiently large to be an effective energy storage device.

Within Inv.1, the two control variables are the modulation index $m$ and the phase angle $\delta_{f}$ of the inverter. By controlling them. Inv.1 front-end voltage $\gamma_{f} \angle \delta_{f}$ as shown in Fig. 4 can be regulated and the power fow $P_{r}+j\left(_{r}\right.$ is therefore controlled. The role of Inv.2 is to ensure a high ac waveform cluality at the terminals of the SP load, again through the control of its modulation index and phase for a given $V_{d}$.

Reference [7] proposed that the DG supply part of the dc and SP load under steady-state. Notice that in the event of a decrease of upstream infeed at point "A," the SOFCDG has to increase its output power instantaneously to satisfy the load demand. However. (10) shows that the SOFCDG has only a limited ability to react instantaneously to a real power change. Hence, the scheme in [7] has to be modified, as follows.

In the scheme proposed here, the SOFCDG supplies the entire power demand of dc and SP loads under the normal steady-state. As the dc and SP load demands vary, the output power of the SOFCDG would vary correspondingly. This load-tracking feature of the SOFCDG is possible, as the expected load variations would be slow compared to the response of the SOFCDG power plant. Clearly, the rating of the SOFCDG will have to be at least that of the maximum of the total dc and SP load demands. This is a reasonable requirement because in the event of a complete loss of the upstream system, the SOFCDG will then be called upon to meet the total demand of the dc and SP loads.

Having met the total dc and SP load demands, the SOFCDG could also inject any surplus active power $\left(P_{r}\right)$ to the PCC bus. $P_{r}$ is then used to meet part of the OQ and HQ load demands. 
Under such an arrangement, the SOFCDG and downstream loads are always connected to the upstream grid. by having Inv.l operating in the inversion mode. The upstream power system therefore acts as a backup source for the SP and de loads in the event of the loss of the SOFCDG. Hence, the SP and dc loads enjoy a supreme level of supply reliability as intended.

While the above operating scenario can be expected to prevail under almost all instances, one has to consider the possibility of the loss of the connection between Inv. 1 and the PCC (i.e.. "B" open). This is a necessary consideration because this incident will result in the output of the SOFCDG being decreased suddenly by an amount equal to $P_{r}$. In order to safeguard the FC stack, the steady-state predisturbance value of $P_{r}$ (i.e., $I_{r .11}$ ) must therefore be constrained to within the range

$$
0 \leq P_{r: 0} \leq|\varepsilon| P_{t y, 0} .
$$

Equation ( 11 ) is obtained as a consequence of (10) so as to satisfy the FOA constraint. However

$$
P_{d y, 0}=P_{(4)}+P_{r .0}
$$

where $I_{S I}^{\prime}$ is the total power demand of the SP and do loads. The output power of SOFCDG $P_{d y .11}$ should also satisfy the output-power limit constraint (4) during steady-state operation. Combining the requirements (11) and (12), one arrives at the condition

$$
(1-|\varepsilon|) P_{d y, 0} \leq P_{S I)} \leq P_{d y(1)}
$$

Yet another steady-state consideration concerns the possibility of reverse power flow into the upstream power system. In the event that the total demand of the HQ and OQ loads is less than $I_{r, 0}^{\prime}$, the surplus portion of $P_{r, 0}$ would flow reversibly back to the upstream system. Perhaps due to contractual reasons, assume such reverse power flow is prohibited. Therefore, it is proposed that active power flow in the upstream feeder (point "A") is continuously monitored. Once reverse power flow is detected, the output power $\left(P_{d q}\right)$ of the SOFCDG is to be reduced until the reverse power flow ceases and (13) is also satisfied.

Apart from supplying active power to the OQ and HQ loads. it is also proposed that Inv.1 is set to supply all of the reactive power required by the OQ and HQ loads under steady-state, that is

$$
Q_{r .0}=Q_{0(2.0}+Q_{1 \mathrm{H}(2.0} .
$$

Power factor at " $\mathrm{A}$ " is therefore maintained at unity. The voltage drop across the equivalent system impedance $(R+j \omega L)$ is therefore minimized. This is desirable as it will ensure efficient power transfer through the upstream system.

Finally, the current rating of Inv.1. denoted as $I_{\text {im } 1, \text { rat } 1 \text {, } 1 \text {, }}$ needs to be examined. Under this normal steady-state condition, Inv. 1 must be rated to supply the proportion of the total maximum HQ and OQ load demands. Thus, by expressing power as the total three-phase quantity and voltage as phase-ground value

$$
I_{\text {in 1,rate }}=\frac{\sqrt{\left(P_{r .0}^{2}+\left(Q_{r .0}^{2}\right)_{\max }\right.}}{\left(3 V_{p, 0}\right)} .
$$

\section{B. Operation Under the Loss of SOFCDG}

Next, consider the loss of all or part of the SOFCDG output power. This incident could be due to an internal fault or maloperation of the FC system. In order to protect the dc and SP Ioads from the impact of the loss of $P_{d y,}$, the upstream system must be forced to supply the additional active power as rapidly as possible to meet the downstream load demands. Therefore, Inv. 1 is to operate in rectifier mode to transfer active power from the upstream system to the do bus.

The most severe condition under this contingency scenario would be the total loss of power from the SOFCDG. The upstream system must have sufficient capacity to supply the maximum de and SP total real power demand $\left(P_{S} P_{\text {m.mix }}\right)$. Hence, one arrives at another Inv. 1 current rating

$$
I_{\text {inv 1.rate. }}=\frac{P_{S D, \text { max }}}{\left(3 I_{p, 0}\right)} .
$$

Combined with (15), the current rating of Inv. 1 is therefore the larger value of $I_{\text {inv } 1, \text { ratel }}$ and $I_{\text {inv1.rate' }}$.

\section{Operation Under Upstream Voltage Sag}

A most devastating voltage disturbance on loads is that of a voltage sag. Due to the complexity and extensiveness of the upstream system, only the balanced voltage sag will be considered and the sag magnitude is assumed constant over the duration of the disturbance. The SP and dc loads could be shielded from the effects of the decrease in the PCC voltage by the corrective actions of Inv.2. However, as the OQ and HQ loads are connected directly to the PCC, they will experience the full impact of the sag. Thus, special consideration must be given to improve the ridethrough capability of the HQ and OQ loads during the voltage disturbance.

Electrical equipment manufacturers often provide information describing the withstand voltage versus time characteristics of their products. A well-known and typical example is the ITI (CBEMA) curve. It relates the duration that a load is capable of withstanding a depressed voltage of a given magnitude. Hence, the more severe a sag is, the shorter the tolerable duration $T$. The curve also indicates the lowest acceptable voltage level $V_{\text {min }}$ for continuous operation of the loads. Suppose the HQ and OQ loads have such voltage/time characteristics. The proposed ridethrough scheme described below will then depend on which of the two scenarios the sag is related to.

Scencrio 1: This scenario pertains to sags that have a duration of less than the specified $T$ for the HQ and OQ loads. Under such a situation, the HQ and OQ loads would not be damaged and they can still be connected to the upstream system. Thus, the system continues to be operated as under the normal steady-state.

Scenario 2: If the sag duration were to exceed $T$, it is proposed that the switch C connected to the OQ load would operate 
at the first available instance to disconnect the $O Q$ load from the network. The OQ load only requires the lowest level of supply quality among all of the loads. Thus, it is chosen to be the first to be disrupted due to the severe sags. After the shedding of the OQ load, if the PCC voltage recovers to above $V_{\text {min }}$, the PQCC would revert to the Scenario 1 control scheme described earlier but without the OQ load. Otherwise, the switch "A" in Fig. 4 in the upstream intercomection link is opened at the earliest possible instant. The PQCC system then operates in an islanding mode.

Although the OQ load can be shed, the HQ load would still be supplied by the PQCC under this islanding stage. The initial power supplied by the SOFCDG to the upstream load $P_{r, 0}$ is unlikely to match the HQ load requirement exactly. Thus, the output power of the SOFCDG has to be regulated subsequent to the islanding. From (10), it is shown that the instantaneous power change imposed on the SOFCDG must be kept at less than $|\varepsilon| I_{t, t_{1}, 0}^{3}$. While Inv. 1 still operates in inversion mode, it is proposed that its role is changed from controlling $P_{r}+. j\left(Q_{r}\right.$. to controlling the PCC bus voltage $\left(V_{p}\right)$ and in maintaining the bus frequency. The objective is to maintain $V_{p}$ at $V_{\text {min. }}$. Hence, following the islanding, the HQ load will draw power $P_{\mathrm{IIQ} \text {, iw }}$ at a level that should be limited to

$$
0 \leq P_{\mathrm{HQ}, i, \ldots, 0} \leq P_{r \cdot 0}+|\varepsilon| P_{i g .(1)}
$$

Combining (17) and (11), the possible power range of the HQ load in the islanding mode can be determined as

$$
\text { () } \leq P_{\mathrm{H}(2, i, \mathrm{~s})} \leq 2|\varepsilon| P_{d g, 0} .
$$

However. as the initial operating condition is governed by (13). it can be combined with (18) to yield

$$
0 \leq P_{H(\text { Riso }} \leq\left(\frac{2|\varepsilon|}{1-|\varepsilon|}\right) \cdot P_{S H)}^{\prime} .
$$

One must also limit the power output of the DG to the range specified by (4), that is

$$
P_{\mathrm{min}} \leq P_{\mathrm{SD}}+P_{\mathrm{H}(2, i)} \leq P_{\mathrm{max}}
$$

It is seen that in order for the power change to be met by the SOFCDG instantaneously and without violating the FC $u$ and the output-power limits, the postfault HQ, SP, and do load levels must comply with (19) and (20). The result of the above derivation can be summarized graphically in Fig. 5. Equation (19) is shown by the boundary line "RS." (20) is shown by the line "ST" and "RU." Clearly, in order for the SOFCDG to meet any instantaneous power change when point " $\mathrm{A}$ " is opened, the only feasible load regime is within the area "RSTUR."

From Fig. 5, the maximum HQ load that can be supplied in islanding mode corresponds to point "S." It can be determined by solving (19) and (20) simultaneously to yield

$$
P_{\mathrm{H}(2, i, \text { so max }}=\frac{2|\varepsilon| P_{\max }^{\prime}}{(1+|\varepsilon|)}
$$

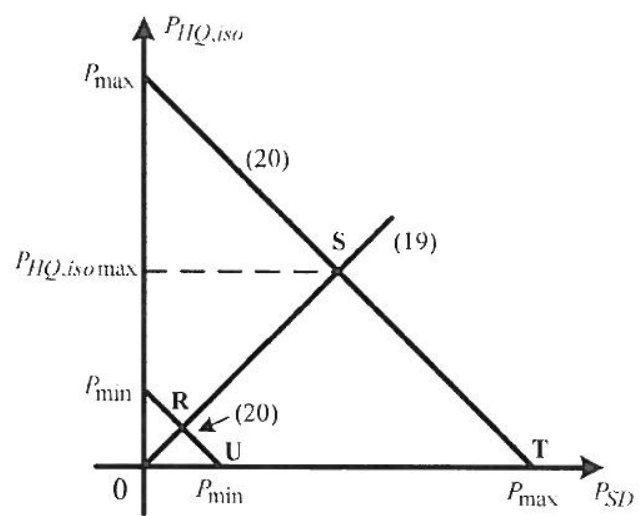

Fig. 5. PQCC leasible operating zone under Scenario 2

TABI.E I

POWIR-QI AI.ITY I.IAELLS OI L.OADS

\begin{tabular}{|c|c|c|}
\hline Loads & Waveform quality & $\begin{array}{c}\text { Voltage sag ride- } \\
\text { through attribute }\end{array}$ \\
\hline OQ & Not improved & Low \\
\hline HO & Not improved & High \\
\hline SP & Very high & Very high \\
\hline DC & Improved & Very high \\
\hline
\end{tabular}

The HQ load in the isolated mode as obtained from Fig. 5 can then be translated to its prefault value by applying

$$
P_{\mathrm{IIQ}, 0}=\frac{P_{\mathrm{HQ}, i \times 0}}{V_{\text {min }}^{\mathrm{a}}}
$$

where $P_{\mathrm{HQ}, 0}$ and $P_{\mathrm{HQ}, i, n}$ denote the prefault and postfault HQ load power demands, respectively, and $\alpha$ is the real power-voltage index [16] of the HQ load. Typically, $0 \leq x \leq 2$.

Based on the above consideration, it can be seen that the loads are to be served by a power-supply system with varied quality levels. Table I summarizes the levels enjoyed by the OQ. HQ, SP, and dc loads under the proposed arrangement.

\section{Controi. Schime.}

The control scheme for the varied PQCC operating conditions is considered next. The interconnected system, which includes the normal steady-state, SOFCDG outage state, and upstream voltage sag (Scenario 1), will be described first.

\section{A. Control Scheme Under Interconnected State}

From Fig. 4, the voltage-current relationship of the ac link between the PCC and Inv. 1 in the $d-q$ frame can be written as

$$
L_{f} \frac{d}{d t}\left[\begin{array}{c}
i_{d l} \\
i_{q}
\end{array}\right]=\left[\begin{array}{cc}
0 & \omega L_{f} \\
-\omega L_{f} & 0
\end{array}\right]\left[\begin{array}{c}
i_{d} \\
i_{q}
\end{array}\right]+\left[\begin{array}{c}
V_{f d}-\sqrt{3} V_{\eta} \\
V_{f_{q}}
\end{array}\right]
$$

where $\left[i_{d}, i_{q}\right]^{T}$ and $\left[V_{f d l}, V_{f q}\right]^{T}$ contain the $d-q$ components of the vectors of the ac-link phase currents $\vec{i}_{f}$ and Inv.1 front-end phase voltages $\vec{V}_{f}$, respectively. It is seen from (23) that cross-coupling effects exist between the state variables $i_{d}, i_{q}$ and the control variables $m, \delta_{f}$. A decoupled control scheme based on [17] was used in this investigation to obtain Fig. 6. in which $i_{d}$ and $i_{q}$ are seen to track the reference 


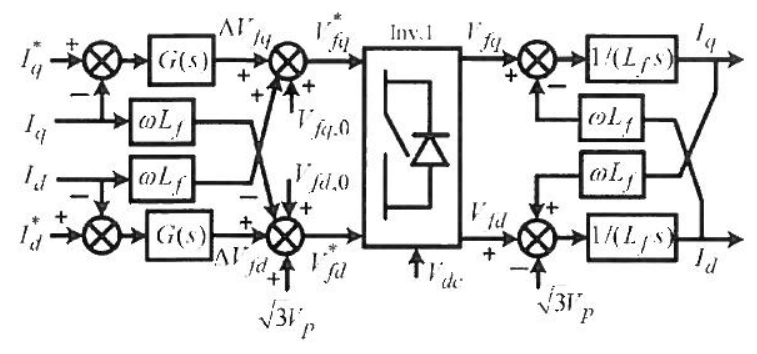

Fig. 6. Control scheme of Inv. Iunder-interconnected state.

currents $i_{i}^{*}$ and $i_{i}^{*}$ separately through the proportional-integral controller $\left(i(s)\right.$. The regulated signals $\left(\Delta V_{f i l}\right.$ and $\left.\Delta V_{f_{q}}\right)$ are then added to the initial voltages $\left(l_{f d .0}\right.$ and $\left.V_{f q, 0}\right)$ to produce the reference voltage signals $\left(V_{f d}^{*}\right.$ and $V_{f_{j}}^{*}$ ). These reference voltages are used to generate the control variables of Inv.1. In designing $(i(s)$, the frequency-response tuning technique has been adopted. As the switching actions of Inv.1 may affect the dynamic performance of the PQCC. it is a good practice to set the bandwidth of the closed-loop system to be from one-fifth to one-half of the switching frequency.

\section{B. Control Scheme Under Islanding Mode (Scenario 2)}

Under Scenario 2, the PQCC is disconnected from the upstream power system. Inv. 1 is to maintain $V_{p}$ at $V_{\text {min }}$ at the rated frequency. Under this islanding mode, the phase angle $\partial_{f}$ is no longer a control variable as the isolated system has only one voltage source connected to the ac link. The control scheme for Inv. 1 is therefore similar to that of Inv.2, details of which are well known (see. for example, [18]).

\section{Determination of Current References}

Having examined the controller design, the current references for the different operation states will now be given.

Under Normal State: Under steady-state. Inv. 1 transfers active power $P_{r, 0}$ from the de bus to the ac system and also supplies all of the reactive power required by the HQ and OQ load. Therefore, the current references are given by

$$
i_{i}^{*}=\frac{P_{r, 0}}{\left(3 \mathrm{~T}_{p, 0}\right)}: \quad i_{i}^{*}=\frac{\left(Q_{() Q .0}+Q_{\mathrm{H}(2,0}\right)}{\left(3 \mathrm{I}_{p, 0}\right)}
$$

Under Sudden Loss of SOFCDG: Under the SOFCDG outage condition. Inv. 1 changes from inversion mode to rectifier mode. Inv. 1 carries the total active power demand of the do and SP loads, with $Q_{r}=0$. Thus

$$
i_{d}^{\times}=-\frac{P_{S D}}{\left(3 V_{p,(1)}\right.}: i_{q}^{*}=0 .
$$

Under Voltage Sag (Scenario 1): Under this scenario, sup-

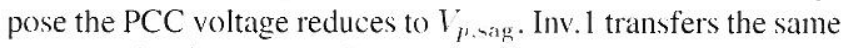
amount of active power $\left(I_{r, 0}^{r}\right)$ as before the sag from the dc bus to the ac system while, at the same time, providing all of the reactive power needs of the OQ and HQ loads. Therefore

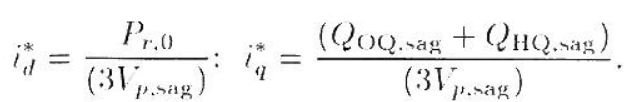

\begin{tabular}{|c|c|c|c|c|c|}
\hline \multicolumn{6}{|c|}{ Power system and Loads: $\omega=5011 \%$} \\
\hline$I_{n ! n}[\mathrm{~V}]$ & 86.6 & $J_{i, u|V|}$ & 33() & $L[\mathrm{mH} H]$ & 0.0358 \\
\hline$R[\Omega 2]$ & $1.36+c-4$ & $c \cdot[u F]$ & 200 & $\left.R_{a} \mid \Omega \Omega\right]$ & 3.63 \\
\hline l. $[\mathrm{mH}]$ & $1.737 \mathrm{e}-3$ & $R,\lfloor\Omega]$ & 0.2205 & $L . .[\mathrm{mIH}]$ & 0.716 \\
\hline$R_{u}[\Omega]$ & 0.1158 & $R_{h}|\Omega|$ & 0.8224 & & \\
\hline$\left.L_{-1} \mid \mathrm{mHH}\right]$ & 0.121 & $L_{\mu}[\mathrm{mH}]$ & 0.86 & & \\
\hline
\end{tabular}

TABI.F. II

PARAMITRIC VALIES Of THL: PQCC ANI) PONFR SYSTLM

Note that in generating the current references, the PQCC loads would vary as part of the normal operating condition. Hence. $i_{i}^{*}$ and $i_{q}^{*}$ will have to be adjusted continuously to track the load changes.

\section{III.USTRATIVF. EXAMPI.ES}

\section{A. Sistem Description}

In order to ascertain the effectiveness of the proposed operating scheme, a series of simulation studies has been performed. The example system used in the studies is as shown in Fig. 4, with the corresponding system data given in the Appendix (Table II). The SOFCDG dynamic model shown in Fig. 2 is incorporated into the PQCC in the simulation in order to improve the accuracy of the study. The SOFCDG rating is $P_{\text {max }}=$ $100 \mathrm{~kW}$ and the relevant parameters used in the simulation were obtained from [12]. The fuel utilization factor under steady-state is assumed to be $\left(0.8\right.$, with $u_{\min }=0.7$ and $u_{\max }=0.9$. From (8) and (10), it is then established that $\Delta P_{d y}^{\prime} \leq 0.125 P_{d g,(1 .}$

The HQ and OQ loads are assumed to be of constant impedance type (i.e., $\alpha=2$ ). Their withstand voltage-time characteristic is assumed to be governed by the ITI curve with $V_{\text {min }}=0.9$ p .11 . Therefore, the maximum HQ load power that can be supported by the PQCC under islanding mode at the minimum voltage can be calculated from (21). viz.. $P_{\mathrm{H}(2,7, \mathrm{~s}) \operatorname{mix}}=22.22 \mathrm{~kW}$. From (22), this corresponds to a maximum prefault power of $P_{1 H(2.0 \text { max }}=27.43 \mathrm{~kW}$. Therefore, in the illustrative example, the initial HQ load power demand could be chosen up to $27.43 \mathrm{~kW}$.

System and load parameters are then calculated according to the following assumed active power distribution: under initial steady-state $P_{\left.d g f_{0}\right)}=90 \mathrm{~kW}, P_{D C}=30 \mathrm{~kW}, P_{S P^{\prime}}=50 \mathrm{~kW}$, $P_{r .0}=10 \mathrm{~kW}, P_{O Q}=175 \mathrm{~kW}$, and $I_{\mathrm{HQ}}=25 \mathrm{~kW}$. The power factor of the OQ and HQ loads is considered as 0.95 lagging. Inv. 1 is represented by six on-off switches with small finite series resistances. The switching frequency of Inv. 1 is 4 $\mathrm{kHz}$.

Inv. I current rating can be determined using the approach described in Section III. In the example, it is seen that

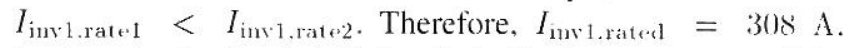
Inv. 1 controller bandwidth is selected to be one-quarter of the switching frequency. The corresponding PI controllers for Inv. 1 when the PQCC is operating under interconnected condition has been determined as $G(s)=0.05+0 .(1) 5 / \mathrm{s}$.

\section{B. Simulation}

Simulation studies have been carried out using Matlab/Simulink to verify the effectiveness of the proposed scheme. Results of four cases are described: that of voltage sag 


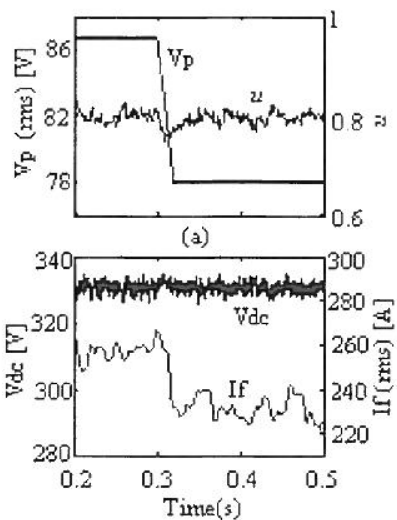

(b)
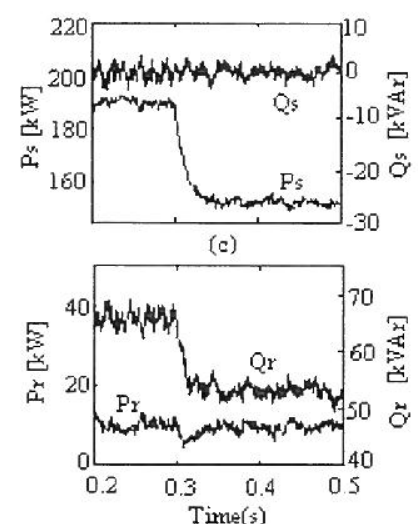

(d)

Hig. 7. System operation under a $90 \%$ voltage sag (scenario 1). (a) PCC voltage (phase a) and utilization factor of SOFCDG. (b) DC-linh voltage and Inv, I current (phase a). (c) Active and reactive power supplied by the upstream source. (d) Active and reactive power supplied by Ins:1.

to $90 \%$ and $60 \%$ of its nominal value. under the sudden loss of the SOFCDG and under the sudden loss of the ac link (CB "B" open). For the SOFCDG. as both the hydrogen input and stack current can be measured on line, 1 can therefore be determined in real time using (3). The corresponding simulation results are shown in Figs. 7-10.

Fig. 7 shows the system response under the $90 \%$ sag condition. Since the HQ and OQ loads are assumed capable of sustained operation at this voltage level, the PQCC system would therefore remain connected to the upstream power system during the sag. Inv. 1 transfers an almost constant $10 \mathrm{~kW}$ throughout the duration of the disturbance. This can be seen in Fig. 7(d). However, due to the voltage dependency of the reactive power demand of $\mathrm{HQ}$ and $\mathrm{OQ}$ loads, $Q_{r}$. supplied by the PQCC is reduced as shown in Fig. 7(d). As the output power of SOFCDG is unchanged, Fig. 7(a) and (b) confirm that the FC fuel utilization factor and the dc-link voltage are undisturbed during the sag. As the real power demand of the HQ and $\mathrm{OQ}$ loads decreases, the power drawn from the upstream power system reduces as shown in Fig. 7(c) while the reactive power from this source remains at zero since the PQCC supplies all of the reactive power required by these loads.

Fig. 8 shows the consequence of a voltage sag $(60 \%)$ for which the PQCC needs to operate in the islanding mode. From the ITI curve, the maximum duration $\left(T^{\prime}\right)$ of the HQ and OQ loads that could tolerate the $60 \%$ sag is about $20 \mathrm{~ms}$. Therefore, in Fig. 8, the OQ load is assumed successfully disconnected by the action of the $\mathrm{CB} 10 \mathrm{~ms}$ after the incipient of the sag. Since the PCC voltage $\left(V_{p}\right)$ remains below $V_{\text {min }}$ after the load shed, the PQCC system is then disconnected from the upstream power system half a cycle later. Thereafter, $P_{s}=Q_{s}=0$ as shown in Fig. 8(c). The operating mode of Inv.1 is changed from controlling the power flow to regulating $V_{p}$ and frequency. Fig. 8(a) shows that $V_{p}$, can indeed be restored to 0.9 p.u. during this islanding mode operation. The SOFCDG output power increases by $10 \mathrm{~kW}$ as evident from Fig. 8(d) and the step power change is within the FOA of the SOFCDG. The SOFCDG fuel utility factor $u_{s}$ increases but does not exceed $u_{\text {max }}$. The dc-link voltage decreases slightly following the islanding stage.

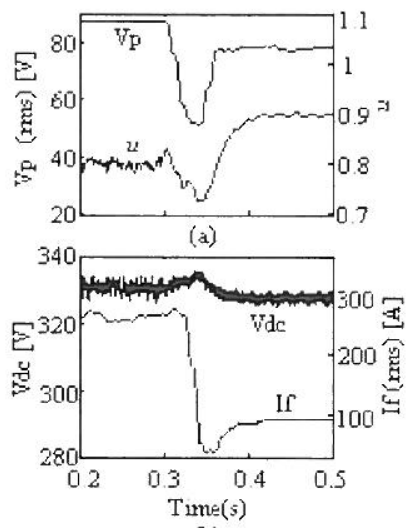

(b)
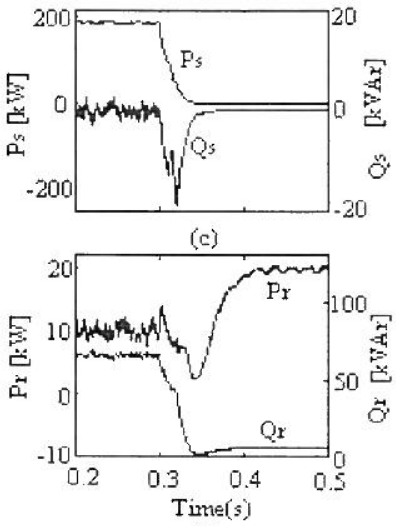

(d)
Fig. 8. System operation under a 6()$^{\circ}$ e voltage sag (scenario 2). (a) PCC voltage (phase a) and utilization factor of SOFCDG. (b) I)C-link voltage and Inv. 1 current (phase a). (c) Active and reactive power supplied by upstream source. (d) Active and reactive power supplied by Ins:1.

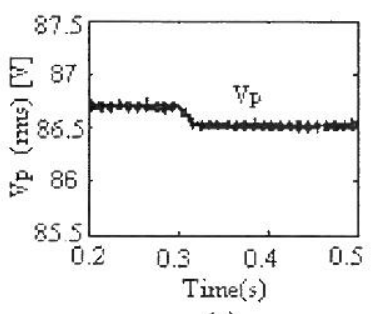

(a)

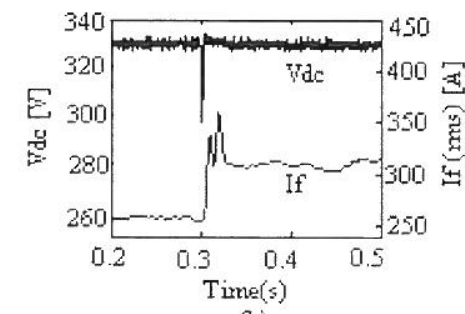

(b)
Fig. 9. System operation under sudden loss of SOFCDG: (a) PCC voltage (phase a). (b) I)C-link voltage and Inv. I current (phase a).

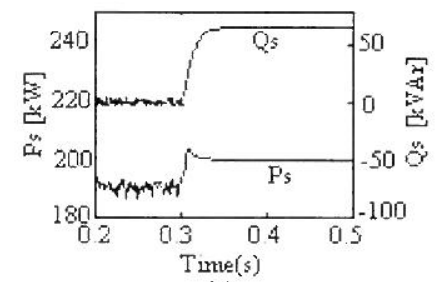

(a)

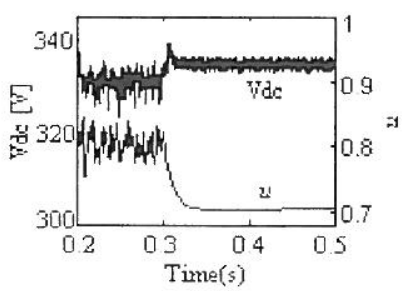

(b)
Fig. 10. System operation under sudden loss of an ac link (CB " $B$ " open). (a) Active and reactive power supplied by an upstream source. (b) DC-link voltage and utilization factor of SOFCDG.

Fig. 9 shows the system response under the sudden loss of the SOFCDG. In order to protect the dc and SP loads, the upstream system has to meet all of the active power load demand rapidly and Inv. 1 is therefore changed from inversion to rectification operating mode. It is seen that $V_{d c}$. decreases at the instance of the DG disconnection. However, it recovers rapidly because of the support from the upstream system. The loads are almost unaffected. Inv. 1 current would increase to its rated value during the fault, as can be seen from Fig. 9(b).

The simulation results under the sudden loss of the ac link (CB "B" open) are given in Fig. 10. Both real and reactive powers supplied by the upstream system shown in Fig. I0(a) increase as the loads are totally supplied by the upstream system. Since $P_{r, 0}$ was chosen to satisfy (11), the sudden power change would not damage the SOFCDG, as can be seen from Fig. 10(b). The utilization factor $u$ is within its limit during the fault event. 
$Y_{\text {de }}$ increases slightly due to the reduced output power of the SOFCDG.

\section{CONCI.USION}

The operation scheme of the PQCC under steady-state, upstream voltage sag. or ac interconnection outage states has been presented. The DG considered is in the form of an SOFC. Unlike previous works on the PQCC. the role of the DG and the inverters in the $\mathrm{PQCC}$ are examined in regards to mitigating the impacts of the fault disturbances on loads. By taking into consideration the ability of the SOFCDG to track load changes, it is proposed that the SOFCDG supplies all of the downstream loads under steady-state. Upstream voltage sag is dealt with in accordance to the severity of the sag. Two possible scenarios have been considered in which the design criteria provide maximum support for load ridethrough while maintaining the unbundled power-supply feature of the PQCC. The proposed scheme exploits more fully the control of the inverters and the SOFCDG to mitigate the voltage-sag effect rather than rely on the use of alternative feeder and the transfer switches, as was suggested in earlier works. Analysis of the power system response and the design of the control system of the PQCC have been also presented. The efficacy of the scheme is born out by simulation results.

\section{REFERENCES}

11] N. Jenkins. Embedded Ge'neration. I.ondon. U.K.: Institution of F.lectrical ringineers. $\mathrm{c} 200(0)$

[2] N. Jenkins and G. Strbac. "Fffects of small embedded generation on power quality." in Proc. Inst. Elact. Eng. Collog. Iswe's in Pencer Quality Conf.. Warwich. U.K.. Nor. 1995. pp. 6/1-6/4.

[3] R. I.asseter and C. Hocheraf. "Unbundled power quality services: Technical issues." in Proc. 1.3th lint. C'ml. Sistem Scicne's, vol. 5. Jan. 1997. pp. $581-588$.

[4] T. Kiichiro. N. Koichi. J. Hasegawa. and T. Oyama. "Flexible, reliable. and intelligent electric energy delivery system: Concepts and perspective." in Pros. American Pencer Conf.. vol. 61-1. Chicago. II. 1999. pp $504-511$.

[5] A. Domijan. A. Montenegro. A. J. F. Keri. and K. F. Mattern. "Simulation study of the world"s firs distributed premium power quality park." IEEE Trums. Poner Dol. pt. 2, vol. 20. no, 2. pp. 1483-1492. Apr. 2005.

[6] J. Hasegawa. "Studier on quality control center for FRIENDS." in Prox: Int. Symp. Energy and Enviremincm, Sessiom on Urban Energy Systems. Oakik. Japan. Jan. 2002. pp. 137-142.

[7] T. Ise. "Functions and conligurations of quality control center on FRIENDS." in Proc. Tramsmission and Distribution Comf. Exhibit. Avia Parific. vol. 1. (Oct. 2002. pp. 59(0-595.

[8] L. J. Blomen and M. N. Mugerwa. Fuet Cell Sirtems. New York: Wiley: 1993

[9] M. W. Ellis. M. R. Von Spakorsky. and D. J. Nelson, "Fuel cell systems. Efficient. flexible energy conversion for the 2 ls century." Proc. IEEE. vol. 89. no. 12. pp. $1808-1818$. Dec. 2001.

[10] J. Larminie and A. Dicks. Fuel Cell System Explained. 2nd ed. New York: Wiley. 20()2

111] Y. H. I.i. S. S. Choi. and S. Rajakaruna. "An analysis of the control and operation of a solid oxide fuel cell power plant in an isolated system." IEEE Trams. Energy Comers. vol. 20. no, 2. pp. 381-387. Jun. 2005.
112| J. Padullés. G. W. Ault. and J. R. McDonald. "An integrated SOFC plant dynamic model for power systems simulation." J. Poner Sontes. vol. 86. no, 1-2. pp. 495-500). Mar. 2000).

[13] S. Krumdieck. S. Page, and S. Round. "Solid oxide fuel cell architecture and system decign for secure power on an unstable grid." J. Penter Sources. vol. 125. no. 1. pp. 189-198. Jan. 20(04.

[14] W. Choi. P. Enjeti. and J. W. Howze. "Fuel cell powered UPS syvems: Design considerations," in Proc. IEEE .34th Annual Comf. Poster Ele'ttromica Specialist, vol. 1. Jun. 20(03. pp. 38()-39().

115] J. Wang and F. \%.. Peng. "Low cost fuel cell converter system for residential power generation." IEEE Trams. Ponder Electrom.. vol. 19. no. 5. pp. 1315-1322. Sep. 20(1).

[16] "IFEF lask force on "load representation for dynamic performance analysis."." HEEE Trems. Ponter Syst. vol. 8. no, 2. pp. 472-482. May $199 \dot{3}$

[17] C. Schauder and H. Mehtal "Vector analysis and control of advanced satic VAR compensators." Proc. In.s. Elect. Eng.. pt. C. vol. 140, no, 4. pp. 266-272. Jul. 1993

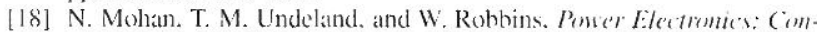
ierters. Applicatioms and Design. 3rd ed: Wiley. 2003.

Y. Q. Zhan received the B.F. degree from $\mathrm{X}_{i}{ }^{*}$ an Jiaotong University. $\mathrm{Xi}_{\mathrm{i}}$ an. China, in 2()()1. She is currently pursuing the Ph.D. degree with Nanyang Technological University. Singapore,

Y. H. Li received the B.E. and M.Eng. degrees from the Huazhong University of Science and Technology. Wuhan. China. in 1994 and 1998., respectively From 1998102001 . he was a Lecturer with Wuhan University. Wuhan. He is currently pursuing the Ph.D. degree with Nanyang Technolegical University. Singapore.

S. S. Choi received the B.E and Ph.D. degrees from the University of Canterbury. Christchurch. New 7ealand. in 1973 and 1976. respectively

He was with the New 7ealand Flectricity Department. Wellingten: National University of Singapore: and the State Energy Commission of Western Australia. Perth. Currently: he is a Profeswor in the School of Flectrical and Electronic Engineering. Nanyang Technological University. Singapore.

S. Rajakaruma received the B.Sc. Eng. degree from the University of Moratuwa Sri lanka. in 1986: the M.Sc. degree from the University of Calgary. Calgary. AB. Canada, in 1989: and the Ph.D. degree from the University of Toronto. Toronto. ON. Canada. in 1993

He was a Senior Lecturer with the Department of Electrical Engineering at the University of Moratuwa from 1993 to 1999. Currently. is an Assistant Profestor in the School of Electrical and Electronic Engineering. Nanyang Technological Universily, Singapore. His research interests include distributed generation. new energy storage techniques, and induction generators.

D. Mahinda Vilathgamuwa received the B.Sc. degree in electric engineering fromt the University of Moratuwa. Sri Lanka, in 1985. and the Ph.ID. degree from Cambridge University. Cambridge. U.K.. in 1993.

Currently, he is an Associate Professor in the School of Electrical and Flectronic Fngineering. Nanyang Technological University. Singapore. Hin research interests are in power quality. control of power converters. and electric drives. 